\title{
¿PUEDE EL UTILITARISMO SER DEONTOLÓGICO? UNA RESPUESTA A KYMLICKA
}

\author{
Hugo Omar Seleme \\ CONICET \\ Universidad Nacional de Córdoba \\ hugoseleme@hotmail.com
}

Resumen: En Teoría de la Justicia, Rawls sostiene que los defectos normativos que contienen algunas concepciones de justicia, como el utilitarismo y el perfeccionismo, se deben a que, por su carácter teleológico, no otorgan prioridad a lo correcto en relación con lo bueno. Kymlicka ha cuestionado esta afirmación siguiendo la estrategia de brindar una interpretación del utilitarismo que respete la prioridad de lo correcto. Esto mostraría que los defectos normativos que Rawls atribuye al utilitarismo no podrían explicarse por su carácter teleológico. La clasificación de las doctrinas en deontológicas o teleológicas carecería de la relevancia que Rawls le adjudica. El presente artículo tiene por objeto mostrar que el argumento de Kymlicka para señalar la trivialidad de la clasificación no se sostiene, pues fracasa en su intento de brindar una interpretación deontológica del utilitarismo.

PALABRAS CLAVE: Rawls, prioridad, correcto, bondad

SUMmary: In his $A$ Theory of Justice, Rawls claims that the defects that some conceptions of justice, such as utilitarianism or perfectionism, have at a normative level are due to the fact that, because of their teleological character, they do not give priority to what is right as opposed to what is good. Kymlicka has questioned this statement. His strategy has been to provide an interpretation of utilitarianism which respects the priority of what is right. This would prove that the defects at a normative level ascribed to utilitarianism by Rawls do not respond to its teleological character. The classification of doctrines into deontological or teleological would lack the relevance that Rawls ascribes to them. This article aims at proving that Kymlicka's argument to point out the triviality of the classification is not successful because he fails to provide a deontological interpretation of utilitarianism.

KEY WORDS: Rawls, priority, right, goodness

\section{Introducción}

Desde la aparición de A Theory of Justice (Rawls 1971), la cuestión referida a la prioridad de lo correcto en relación con lo bueno ha sido uno de los principales criterios para clasificar 
las teorías políticas. En efecto, en esta obra, Rawls sostiene que los principales defectos que padecen teorías como el utilitarismo y los distintos tipos de doctrinas perfeccionistas se deben a una falla en su estructura que consiste en no otorgar esta prioridad. Por el contrario - afirma Rawls-, estas concepciones ven lo correcto como aquello que maximiza el bien, definido de manera independiente.

En A Theory of Justice, las teorías con esta estructura se denominan "concepciones teleológicas". Caracterizando a este tipo de doctrinas, Rawls señala:

Los dos conceptos principales de la ética son aquellos de lo correcto y lo bueno [...]. Entonces, la estructura de una teoría ética está determinada, en gran parte, por el modo en que define y conecta estas dos nociones básicas. Ahora bien, parece que la manera más simple de relacionarlas es la adoptada por las teorías teleológicas: el bien se define independientemente de lo correcto, y luego lo correcto se define como aquello que maximiza el bien. (Rawls 1971, p. 24 [21-22]) ${ }^{1}$

En línea con lo señalado, Rawls sostiene que una de las principales virtudes de cualquier concepción de justicia $-\mathrm{y}$ de la suya en particular - es su carácter deontológico; esto es, el otorgar prioridad a lo correcto con respecto a lo bueno. La definición de una doctrina deontológica se determina por oposición a la idea de doctrina teleológica. Así, una teoría deontológica es aquella que:

o bien no especifica lo bueno independientemente de lo correcto, o bien no interpreta lo correcto como la maximización de lo bueno. (Habría que hacer notar que las teorías deontológicas se definen como no teleológicas, no como posturas que caracterizan la corrección de las instituciones y las acciones, independientemente de sus consecuencias. Todas las doctrinas éticas que merecen nuestra atención toman en cuenta las consecuencias al juzgar la

${ }^{1}$ Los números entre corchetes corresponden a la edición revisada de 1999. Las traducciones son del autor. 
corrección. Si alguna no lo hiciera, simplemente sería irracional, desquiciada.) (Rawls 1971, p. 30 [26])

De este modo - según la exposición que del tema hace Rawls en $A$ Theory of Justice-, las discrepancias que en el nivel normativo mantiene su propia concepción de justicia con las concepciones utilitaristas y perfeccionistas se deben a una profunda divergencia estructural en el nivel metaético.

Desde la publicación de la obra de Rawls, clasificar las concepciones de justicia en teleológicas y deontológicas ha pasado a ser una tarea de especial relevancia para todo teórico que pretenda comprender el particular contenido normativo de cualquiera de ellas. Porque si el carácter teleológico o deontológico de una concepción es — como sostiene Rawls - la raíz profunda de sus vicios y virtudes en el nivel normativo, entonces, cualquier intento de entender cabalmente sus conclusiones en ese nivel debe ocuparse también de desentrañar su estructura metaética.

Will Kymlicka ha sido quien con más énfasis ha cuestionado esta tesis rawlsiana en un artículo aparecido en Philosophy and Public Affairs (Kymlicka 1988). Su estrategia ha consistido en mostrar que entre Rawls y sus opositores, en particular los utilitaristas, no existe discrepancia respecto a si lo correcto es prioritario en relación con lo bueno. Manteniendo sus divergencias en el dominio normativo, el utilitarismo y la justicia como equidad pueden interpretarse como concepciones deontológicas. Esto serviría para mostrar que no es posible explicar tales divergencias basándose en el hecho de que la concepción otorgue o no prioridad a lo correcto con respecto a lo bueno. Contrariamente a lo que sostiene Rawls, se habría mostrado que los supuestos defectos que el utilitarismo padece en el nivel normativo no pueden explicarse por una falla estructural en el nivel metaético. De esta manera, la clasificación propuesta por Rawls entre teorías teleológicas y deontológicas sería menos fecunda de lo que éste piensa cuando se trata de entender las teorías políticas contemporáneas. ${ }^{2}$

${ }^{2}$ Afirma Kymlicka: "El problema de si lo correcto o lo bueno es prioritario se concibe ahora como un punto fundamental para dividir las teorías políticas 
Por supuesto, Kymlicka no niega que exista una posible interpretación teleológica del utilitarismo; y ello no es necesario para que su estrategia tenga éxito. Su objetivo es más modesto. Consiste, simplemente, en demostrar que, en contra de lo que sostiene Rawls, es posible una interpretación deontológica de esta concepción política.

Si esto es así, concluye, no es cierto que las deficiencias que en el nivel normativo presenta el utilitarismo se expliquen por su carácter teleológico. Se podría brindar una interpretación deontológica de esta concepción, con sus mismas exigencias normativas, lo que daría sustento, finalmente, a su tesis de que la clasificación de las doctrinas políticas en teleológicas y deontológicas es menos relevante de lo que - a partir de Rawls - han sostenido los filósofos políticos.

Para entender el tipo de argumento que presentaré en contra de Kymlicka, es importante tener en mente que la crítica esgrimida por éste ha sido formulada como una objeción interna a la justicia como equidad; es decir, Kymlicka acepta las nociones de doctrina deontológica y teleológica utilizadas por Rawls y, a partir de éstas, pretende mostrar que es posible brindar una interpretación deontológica del utilitarismo.

Mi argumento está dirigido a demostrar que el intento de Kymlicka de presentar una interpretación del utilitarismo como una concepción deontológica, en el sentido en que Rawls interpreta este término, no tiene éxito. Me propongo poner de manifiesto de qué manera, aun en la interpretación ofrecida por Kymlicka, el utilitarismo sigue siendo una concepción teleológica. Finalmente, esto serviría para quitarle sustento a la tesis de Kymlicka sobre la falta de relevancia de la clasificación de las doctrinas en deontológicas o teleológicas. Ya no podría tomarse como prueba de la misma el hecho de que sea posible dar una interpretación deontológica del utilitarismo, manteniendo sus mismas deficiencias en el nivel normativo.

contemporáneas. [...] Espero poner en duda esta postura. No creo que exista una dificultad real en cuanto a si lo correcto o lo bueno es prioritario" (Kymlicka 1988, p. 173). 
En consecuencia, el objetivo del presente trabajo no es reunir consideraciones que sirvan para fundar la aseveración rawlsiana de que la estructura deontológica o teleológica de una concepción política es una de las principales causas de sus defectos en el nivel normativo; sino, simplemente, desarticular el argumento esgrimido por Kymlicka en contra de esta tesis.

\section{Utilitarismo deontológico y teleológico}

El eje del argumento de Kymlicka es su afirmación de que existen dos interpretaciones posibles del utilitarismo: una deontológica y otra teleológica. Analicemos lo que dice de cada una de ellas.

En su interpretación deontológica — sostiene Kymlicka-, el utilitarismo es un procedimiento para sumar intereses y deseos individuales con el objeto de realizar elecciones sociales (Kymlicka 1988, p. 176). Su principal interés estriba en tratar a las personas como iguales, de allí su carácter de teoría moral (p. 177). Esta premisa básica también funge como tal en la teoría de la justicia como equidad; sin embargo, ambas teorías se distancian en lo que consideran la forma más adecuada para alcanzar ese objetivo. El utilitarismo, a diferencia de la concepción rawlsiana de justicia, plantea que las personas reciben igual consideración y respeto si se da el mismo peso a los intereses de cada una de ellas, sin importar cuál sea el contenido o el portador de la preferencia. Esto, como subproducto del deseo de tratar a los individuos como iguales y sumar sus preferencias imparcialmente, es lo que conduce a maximizar la utilidad (pp. 178-190). ${ }^{3}$

\footnotetext{
${ }^{3}$ Aunque Kymlicka se refiere a preferencias, no es necesario entender que su argumento está dirigido exclusivamente al utilitarismo que interpreta de este modo la utilidad. Tanto lo que dice su argumento, como lo que yo voy a señalar en su contra, puede extenderse al utilitarismo de los estados mentales o de las preferencias, en todas sus variantes. Por otra parte, considero que ninguna de estas versiones es superior a la otra; ambas han sido sometidas a objeciones igualmente devastadoras. Así como Nozick se encargó de presentar un caso hipotético en contra del utilitarismo de los estados mentales en Anarchy, State and Utopia (Nozick 1974, pp. 42-43), Parfit lo hizo contra el
} 
No obstante - continúa Kymlicka-, el utilitarismo sería una concepción deontológica, porque no definiría lo correcto en términos de maximizar el bien - particularmente la utilidad-, sino de respetar el derecho de las personas a ser tratadas como iguales. La maximización de la utilidad es una mera consecuencia de la aspiración de tratar a las personas de esta manera. Al igual que la teoría de la justicia como equidad, el utilitarismo - según esta interpretación - buscaría desarrollar un ideal de imparcialidad o equidad, y en este sentido tendría carácter deontológico (Kymlicka 1988, p. 178).

En su interpretación teleológica — afirma Kymlicka-, el utilitarismo no establece, como premisa básica, el deber de tratar a las personas como iguales, sino el deber de provocar determinados "estados de cosas" considerados valiosos. La acción correcta es aquella que maximiza el "estado de cosas" valioso. Ahora bien, dada la particular concepción que tiene de tal "estado" - entendido como aquel en el que existe mayor bienestar por parte de los individuos-, el utilitarismo postula que el mejor procedimiento para alcanzar este objetivo consiste en contar la satisfacción de los intereses de cada uno como si tuviesen igual peso. Por otro lado, este proceder genera - como subproducto - que se traten los intereses de las personas con equidad.

Así, afirma Kymlicka:

La segunda interpretación le da la vuelta a la primera. La primera define lo correcto en términos de tratar a las personas como iguales, lo que conduce al procedimiento de conteo utilitarista, el cual resulta que maximiza lo bueno. La segunda define lo correcto en términos de maximizar lo bueno, lo que conduce al procedimiento de suma utilitarista, el cual, como una mera consecuencia, trata por igual los intereses de las personas. (Kymlicka 1988, p. 180)

En la primera versión del utilitarismo, el argumento que lo justifica estaría compuesto por las siguientes premisas:

utilitarismo de las preferencias en Reasons and Persons (Parfit 1984, pp. 496497). 
a) Las personas tienen el derecho a ser tratadas como igualmente importantes.

b) Respetar la importancia de las personas implica tomar en consideración los intereses de todos por igual, a partir de lo cual se extrae la siguiente conclusión:

c) Las acciones moralmente correctas son aquellas que desarrollan al máximo la utilidad.

Ésta sería — según Kymlicka - una teoría deontológica, porque lo correcto se define a partir del derecho de cada persona a ser tratada como igual a las demás. El procedimiento de conteo utilitarista aparece como un modo de especificar este derecho con objeto de que permita guiar la acción, y la maximización de la utilidad aparece como un resultado de este procedimiento.

En la segunda versión del utilitarismo, su premisa básica es que se debe obtener un determinado "estado de cosas" valioso, y añade la premisa de que este "estado de cosas" consiste en el mayor bienestar o "utilidad" de los individuos. En última instancia, esto lleva a tomar en consideración el bienestar de todos los individuos y a tratarlos como igualmente importantes, con el fin de maximizar la utilidad.

A diferencia de la primera versión, ésta sería - sostiene Kymlicka - una concepción teleológica. La razón es que aquí lo correcto se define en función de la maximización de un determinado "estado de cosas" considerado valioso o bueno.

Antes de evaluar la afirmación de Kymlicka de que sí existe una posible interpretación deontológica del utilitarismo, sería útil precisar algunos de los conceptos que utiliza Rawls. Esto facilitará la tarea de juzgar adecuadamente si la crítica interna que Kymlicka le formula es pertinente o no.

Un primer punto que se debe dilucidar es a qué tipo de utilitarismo se está refiriendo Rawls en $A$ Theory of Justice. ¿Se está refiriendo específicamente al utilitarismo como criterio de corrección, o como un tipo de procedimiento que garantiza el logro del resultado correcto? En el primer caso, lo que vuelve correcto un determinado acto, o regla, es satisfacer el criterio 
utilitarista. En el segundo caso, la corrección del acto, o regla, viene dada por su conformidad con un principio distinto del utilitarista; y el procedimiento de conteo utilitarista aparece meramente como un mecanismo que permite alcanzar el resultado correcto. ${ }^{4}$

Es evidente que Rawls está interesado en el primer tipo de utilitarismo. Así, la doctrina a la que dirige su crítica es la que sostiene que una sociedad justa es aquella cuyas "principales instituciones están organizadas para alcanzar el mayor saldo neto de satisfacción sumados todos los individuos pertenecientes a ella" (Rawls 1971, p. 22 [20]).

En las referencias de Rawls al utilitarismo - y, en consecuencia, al carácter teleológico que le atribuye-, aquel debe interpretarse como un criterio último de corrección. Lo que a Rawls le interesa es encontrar estándares que establezcan qué es lo correcto; y, para defender los esquemas que él propone, su estrategia es contraponerlos al principio utilitarista. Ahora bien, una de las restricciones del concepto de "lo correcto", según Rawls, es la "definitividad".

Y él señala al respecto:

La quinta y última condición es la de definitividad. Las partes van a considerar al sistema de principios como el tribunal de apelación definitivo en materia de razonamiento práctico. No existen criterios más altos a los cuales se puedan dirigir los argumentos en apoyo de demandas. (Rawls 1971, p. 135 [116])

Un utilitarista, por lo tanto - en el sentido en que Rawls interpreta este término-, es quien sostiene que el principio de utilidad pertenece al código moral de la sociedad. Esto es, alguien que afirma que, dentro de la moralidad pública, el tribunal final de apelación es la utilidad. ${ }^{5}$ No existe otro criterio por encima del de maximización de la utilidad, ni junto a él,

${ }^{4}$ Para ahondar en la distinción entre utilitarismo como criterio de corrección y como procedimiento decisorio, cfr. Brink 1986.

${ }^{5}$ Cfr. Rawls 1971, p. 182, n. 31 [159, n. 32]. 
que sirva para determinar la corrección de un acto o arreglo institucional.

Una segunda precisión atañe a la manera en que Rawls entiende la prioridad de lo correcto con respecto a lo bueno. Para aclarar esta cuestión, es necesario distinguir entre dos maneras diferentes, aunque vinculadas, en las que, según Rawls, se puede cumplir el otorgamiento de esta prioridad.

La primera se refiere a la forma de justificar los criterios de corrección o principios de justicia. Aquí la prioridad se alcanza siempre que en la argumentación que conduce a los principios no se utilicen nociones de bondad que hayan sido definidas con independencia de las exigencias de corrección. No se trata de obtener los principios de corrección sin utilizar ninguna idea de "bien". 6 Esto es imposible, ya que en la argumentación siempre será necesario un criterio que sirva para determinar aquellos frutos de la cooperación social que se consideran valiosos. No se puede argumentar a favor de principios de justicia distributiva, sin tener una idea de los bienes o las ventajas que se van a distribuir. Se trata, en cambio, de utilizar una noción de "bien" configurada a partir del interés por los otros; una noción de "bien" que no sea independiente de las exigencias de corrección.

Así, por ejemplo, cuando Rawls utiliza el recurso de los bienes primarios en el argumento que conduce a sus principios, dicho uso respeta esta restricción. La lista de principios se conforma a partir de la concepción normativa de persona, dotada de sus dos poderes morales. A su vez, esta concepción representa la manera en que, según nosotros, deberíamos vernos al momento de decidir qué reglas van a normar nuestro trato recíproco; plasma la idea de que creemos valioso tratarnos con

${ }^{6}$ Aquí es útil realizar una pequeña digresión para aclarar una idea que puede prestarse a confusión. Una teoría deontológica no sostiene que lo correcto y lo bueno sean conceptos contrapuestos, de modo que nada pueda ser correcto y bueno a la vez. Lo que señala es que son conceptos independientes, de manera que no es suficiente saber que algo es correcto para afirmar que es bueno, ni viceversa. Para percatarse de que esto es así, basta advertir lo siguiente: si fuesen conceptos contrapuestos, toda la empresa de Rawls dedicada a demostrar la estabilidad de su concepción - y la congruencia entre lo correcto y lo bueno- sería inútil. Si, por otro lado, no fuesen conceptos independientes, tal empresa sería innecesaria. La congruencia estaría dada por definición. 
reciprocidad, como si fuésemos seres dotados de autonomía. Es decir, la concepción normativa de persona establece aquello que juzgamos valioso y digno de respeto en nuestro trato con los demás. ${ }^{7}$

La segunda manera de satisfacer esta prioridad de lo correcto ya no se vincula, como la anterior, al argumento que conduce al criterio de corrección, sino al criterio de corrección mismo. Si este criterio o principio establece que la organización institucional correcta es aquella que promueve algo que se considera bueno con independencia de cualquier restricción moral, entonces, la prioridad de lo correcto en relación con lo bueno no se ha cumplido. Lo que es correcto, lo que es debido a los otros, no establece restricciones sobre lo que debe ser promovido, en cuanto sea valioso. Por el contrario, la acción debida es aquella que promueve o maximiza algún objetivo concebido como valioso con independencia de cualquier exigencia moral o consideración vinculada a los demás. ${ }^{8}$

${ }^{7}$ En el mismo sentido, Scanlon sostiene: "estas concepciones de bienestar serán de índole moral; es decir, tales concepciones derivan su significado, y hasta cierto punto su distintiva configuración, del papel que desempeñan en las estructuras morales en las cuales figuran" (Scanlon 1998, p. 110; las cursivas son mías).

${ }^{8}$ Quiero hacer una aclaración con respecto a la noción de valor que estoy utilizando. No debe interpretarse en el sentido de que sólo lo bueno es valioso. La concepción normativa de persona, sobre cuya base se determina la manera correcta de tratar a los otros, también expresa valores, como ya lo señalé.

La diferencia entre las teorías teleológicas y las deontológicas es, entonces, la siguiente. Según las primeras, existe un único dominio de valores, a saber, aquello que debe ser promovido. Lo correcto se define como la acción o el orden institucional que maximiza el valor así entendido. En cambio, para las teorías deontológicas, existen al menos dos dominios de valores. El valor que subyace en lo correcto no es el de la maximización de lo bueno, sino el interés del por los otros. Se considera valiosos a los otros. Satisfacer este valor no consiste en maximizarlo ni en promoverlo, sino en respetarlo. Existen, en consecuencia, dos tipos de comportamientos valiosos y dos tipos de exigencias de conducta: promover el bien y respetar a los demás. Las segundas exigencias limitan a las primeras.

Con estas aclaraciones pueden realizarse algunas precisiones. En primer lugar, para una teoría deontológica, actuar correctamente es algo valioso, aunque lo correcto no consista en maximizar el valor. En segundo, que exista una sociedad regulada por principios correctos de justicia también se considera 
$\mathrm{Si}$, por el contrario, el principio no establece que se debe maximizar ${ }^{9}$ lo bueno definido de forma independiente, entonces se ha cumplido la prioridad de lo correcto.

Lo antes señalado se vincula al concepto de doctrina deontológica; veámoslo con cierto detenimiento. Una doctrina deontológica es aquella que no tiene carácter teleológico. Una doctrina teleológica, por su parte, es aquella que, en primer lugar, define lo bueno con independencia de cualquier exigencia moral y, en segundo, considera que lo correcto es aquello que maximiza el bien así definido. En consecuencia, hay dos formas en que una doctrina puede evitar ser teleológica. La primera consiste en no utilizar una noción independiente de "bien"; la segunda consiste en no proponer un principio maximizador como criterio de corrección. Éstas son, respectivamente, las dos maneras en que, según lo he señalado, se puede cumplir la prioridad de lo correcto.

Así, por ejemplo, un principio que estableciera que lo correcto es distribuir de manera igualitaria aquello que se considera bueno, en cuanto no es un principio maximizador, tendría ca-

valioso. Esto último debe entenderse en dos sentidos distintos, vinculados cada uno de ellos a los dos dominios de valor. La existencia de una sociedad bien ordenada es valiosa, porque permite que los ciudadanos promuevan sus propias concepciones del bien, y porque trata a todos los individuos con igual respeto. Surge aquí, sin embargo, otra dificultad en relación con las teorías teleológicas, que deliberadamente he dejado de lado: ¿acaso la única manera de tratar con un valor que debe ser promovido es maximizarlo? Dada la definición de teoría teleológica propuesta por Rawls, parece que su respuesta se inclinaría a ser afirmativa. Otros, como los consecuencialistas "satisfaccionistas", piensan lo contrario (Slote 1986). Si éstos estuvieran en lo correcto, una teoría completamente "satisfaccionista", y, por lo tanto, no maximizadora, sería deontológica según la definición propuesta por Rawls; no obstante, lo correcto se definiría sin tener en consideración ningún interés moral, es decir, ninguna consideración por los otros. Para una crítica correcta — a mi juicio — de la posición "satisfaccionista", véase Pettit 1997.

${ }^{9} \mathrm{Si}$ el principio no ordena simplemente maximizar el bien, sino que contiene consideraciones en relación con su forma de distribución, entonces — con independencia de si el bien ha sido o no definido de forma independiente - tal doctrina es deontológica, porque no considera que lo correcto sea maximizar el bien. Lo mismo sucedería si el principio ordena maximizar el bien, pero éste no ha sido definido con independencia de cualquier consideración por los otros. 
rácter deontológico. Esto sería así sin importar si el "bien" al que se refiere el principio se ha definido con independencia de cualquier exigencia moral o interés por los otros.

Para hacer más concreto el ejemplo, supongamos que la teoría en cuestión es un tipo de perfeccionismo según el cual el desarrollo de las excelencias humanas, digamos en el ámbito artístico, es bueno en sí mismo, no sobre la base de un interés por los individuos, sino, simplemente, porque se considera que el arte es un valor intrínseco. Si esta teoría no hiciera más que establecer que lo correcto es alcanzar el nivel más alto posible de logros artísticos, sería una teoría teleológica, pues, en la argumentación que conduce al principio de corrección, utiliza una noción independiente de "bien", y el principio de corrección mismo es maximizador. Por lo tanto, la prioridad de lo correcto no se ha cumplido aquí en ninguna de sus dos formas.

$\mathrm{Si}$, por el contrario, la teoría estableciera que lo correcto es lograr que cada individuo alcance, en la medida de sus posibilidades, algún logro artístico, la noción de "bien" utilizada seguiría siendo independiente de cualquier consideración moral o interés por los otros, pero el principio incluiría en su configuración el interés por los demás. Esto se debe a que no exige simplemente que el "bien" sea promovido o maximizado, sino también que sea distribuido de una manera particular. En este caso, la prioridad de lo correcto se habría cumplido por la forma de configurar el principio.

En consecuencia, existen dos maneras de asegurar la prioridad de lo correcto con respecto a lo bueno: una está vinculada a la justificación de los principios; y otra a su configuración. En lo sucesivo, y para simplificar la exposición, denominaré, a cada uno de estos mecanismos, el recurso justificatorio y el configurativo, respectivamente.

Esas dos maneras de satisfacer la prioridad de lo correcto no son equivalentes, y esto se hace patente cuando se toma conciencia de que puede darse el supuesto de que una concepción de justicia no emplee el recurso justificatorio, aunque sí el configurativo. Tal sería el caso, recién expuesto, del principio perfeccionista que exige la distribución igualitaria. 
Lo mismo sucedería con una teoría de tipo intuicionista que tuviera dos principios: uno que sostuviera que las instituciones básicas deben ser diseñadas para producir el máximo saldo neto de satisfacción, y otro que sostuviera que se debe distribuir la satisfacción de manera equitativa. En este caso, si el argumento que conduce al primer principio ha utilizado la noción de satisfacción - la cual se define con independencia de cualquier consideración moral-, tal concepción no habrá utilizado el recurso justificatorio para satisfacer la prioridad de lo correcto en relación con lo bueno. Sin embargo, sí respeta la prioridad, gracias a la utilización del recurso configurativo, puesto que lo correcto — con el agregado del segundo principio- establece restricciones sobre la manera de maximizar las satisfacciones.

A partir de lo explicado pueden extraerse las siguientes conclusiones: para que una teoría tenga carácter deontológico basta que utilice alguno de los dos recursos señalados. En consecuencia, una teoría teleológica es aquella que no echa mano a ninguno de estos recursos, debido a que una teoría teleológica es aquella que no tiene carácter deontológico.

No basta corroborar, entonces, que la teoría no utiliza el recurso justificatorio, o el configurativo, para poder afirmar su carácter teleológico. Es necesario corroborar que no emplea ninguno de los dos. De modo que si se ha determinado que la teoría usa una noción independiente de "bien", todavía no puede afirmarse su carácter teleológico. Es necesario comprobar, además, si su principio es o no un principio maximizador. En el primer caso, la doctrina será teleológica, pero no en el segundo; y lo mismo a la inversa. Aunque se haya determinado que el principio de una doctrina es maximizador, todavía no puede afirmarse su carácter teleológico. Es necesario confirmar, además, si en la justificación del principio se ha utilizado o no una noción de "bien", que sea independiente de cualquier consideración por los otros. En el primer caso, la doctrina será teleológica; en el segundo, no.

Hechas estas precisiones, estamos en condiciones de determinar qué entiende Rawls por concepción teleológica. Según él, es aquella que no considera que lo correcto sea prioritario 
con respecto a lo bueno y, en consecuencia, no utiliza ninguno de los dos recursos para satisfacer dicha exigencia. Es aquella donde la argumentación que conduce a los principios especifica lo bueno con independencia de cualquier exigencia moral, y el principio de corrección obtenido a través de este argumento establece que se debe maximizar el "bien" así especificado; es decir, si una concepción determina que se debe maximizar el "bien", pero en la argumentación que conduce al principio de corrección lo bueno ha sido especificado respetando la prioridad de lo correcto, tal concepción es deontológica.

Lo mismo sucedería si en el proceso de justificación se ha usado una noción de "bien" definida con independencia de cualquier exigencia moral, pero el principio de corrección al que se arriba no determina que lo que se debe hacer es meramente maximizar el "bien", sino que establece restricciones a la promoción de éste, fundadas en la consideración por los otros.

La concepción de justicia rawlsiana echa mano a ambos recursos para cumplir la prioridad de lo correcto en relación con lo bueno. Esto obedece a que, por un lado, en la justificación de los principios de justicia se utiliza una noción no independiente de bienes primarios o teoría restringida del bien, y, por otro, a que los principios de justicia están configurados de tal manera que lo correcto es maximizar la porción de bienes primarios que reciben los menos favorecidos. Lo anterior significa que si bien existe un criterio maximizador, lo correcto no es visto como la maximización de una noción de "bien" independiente. Esta aseveración se fundamenta en dos razones: en primer lugar, que el "bien" al que se hace referencia no ha sido definido de manera independiente; en segundo, que lo correcto no es simplemente maximizar el "bien", sino maximizar la porción de "bien" que recibirían en la distribución los menos favorecidos ${ }^{10}$ una vez que se hayan garantizado de manera prioritaria las libertades básicas iguales de todos los ciudadanos. Dicho de otra manera, el criterio de corrección es un principio maximizador, pero

\footnotetext{
${ }^{10}$ Estas dos razones son, en líneas generales, similares a las que Thomas W. Pogge encuentra para diferenciar la justicia como equidad del utilitarismo. Cfr. Pogge 1989, pp. 36-47.
} 
sensible a la distribución. En consecuencia, lo correcto no se ve como la maximización del "bien" definido de forma independiente, porque, por un lado, la noción de "bien" utilizada por Rawls no tiene esta característica; y, por otro, porque los principios de justicia, aunque expresan la preocupación por la maximización del "bien", también expresan inquietud por su distribución, y éste es un problema vinculado a la preocupación por los otros y, por ende, a lo correcto, no a lo que se considera bueno o valioso. ${ }^{11}$

Rawls emplea de dos maneras el recurso configurativo para garantizar la prioridad de lo correcto con relación a lo bueno: la primera de ellas, al establecer en el primer principio de justicia la exigencia prioritaria de asegurar las libertades básicas iguales de todos los ciudadanos; la segunda, al exigir —en el principio de la diferencia - que se maximice la porción de bienes primarios, distintos de las libertades básicas, que recibirán los menos favorecidos. Ambas exigencias están vinculadas a la distribución y a la preocupación por el otro, es decir, a lo correcto.

Con todas estas precisiones a la mano, es posible evaluar el argumento de Kymlicka a favor de la interpretación deontológica del utilitarismo. Según Kymlicka, existen dos razones para defender esta posible interpretación. La primera hace referencia a la existencia de una manera posible de argumentar a favor de este principio que no parte de una noción de "bien" independiente de cualquier exigencia moral. La segunda se refiere a que existe una forma de interpretar el principio utilitarista, como si éste estableciese restricciones sobre el bien que ha de ser promovido, caracterizando dicho bien con independencia de cualquier restricción moral.

${ }^{11} \mathrm{Al}$ respecto, señala Rawls: "Mientras que si la distribución de bienes también se considera un bien, tal vez de orden superior, y la teoría nos conduce a producir el mayor bien (incluyendo, entre otros, el bien de la distribución), entonces ya no tenemos una visión teleológica en el sentido clásico. El problema de la distribución cae bajo el concepto de lo correcto tal como uno lo entiende intuitivamente, y de este modo la teoría carece de una definición de lo bueno que sea independiente" (Rawls 1971, p. 25 [22]). 


\section{Justificación deontológica del principio utilitarista}

Kymlicka parece creer que alcanzar o no la prioridad de lo correcto, a través de la justificación que se brinda de los principios de corrección, depende exclusivamente del carácter de la premisa básica a partir de la cual se desarrolla el argumento que conduce a ellos. Si la premisa básica es la consideración de un objetivo como valioso, entonces la prioridad no se ha cumplido y, por ende, la concepción es teleológica. Por el contrario, si la premisa básica es un derecho, entonces la prioridad se ha cumplido y la concepción es deontológica. Esto le permite concluir que sólo una de las interpretaciones del utilitarismo es teleológica: aquella en la cual la premisa básica a partir de la que se justifica el principio utilitarista sostiene que determinados estados de cosas - en los cuales se ha producido utilidad - son valiosos. La otra interpretación, por el contrario, sería deontológica. Aquí, la premisa básica para justificar el principio utilitarista es el derecho de las personas a ser tratadas con igual respeto y consideración.

Esta manera de entender el carácter deontológico o teleológico de una doctrina, según la naturaleza de la premisa básica a partir de la cual se justifican sus principios de corrección, fue propuesta por primera vez en Taking Rights Seriously (Dworkin 1977); sin embargo, como reconstrucción del pensamiento de Rawls me parece equivocada. ${ }^{12}$

Tal reconstrucción es errónea por dos razones.

En primer lugar, porque considera que basta determinar si se ha utilizado o no el recurso justificatorio, para establecer el

${ }^{12}$ Dworkin propone refinar la distinción de Rawls y establece tres posibles tipos de teorías políticas: las fundadas en objetivos, las fundadas en un derecho o las fundadas en un deber. Igualmente sostiene que el calificativo de teoría deontológica debería aplicarse sólo a las concepciones pertenecientes al segundo grupo. Señala: "una teoría podría fundarse en un objetivo, en cuyo caso tomaría algún objetivo como fundamental, por ejemplo, promover el bienestar general; podría fundarse en un derecho, en cuyo caso tomaría algún derecho como fundamental, por ejemplo, el derecho de todos los hombres a la más amplia libertad posible; o podría fundarse en un deber, y tomar como fundamental algún deber, por ejemplo, obedecer la voluntad de Dios tal como se expresa en los Diez Mandamientos" (Dworkin 1977, pp. 171-172). 
carácter deontológico o teleológico de una concepción de justicia. Esto es incorrecto ya que si bien es suficiente determinar que se ha usado tal recurso justificatorio para poder afirmar que la concepción tiene carácter deontológico, por otra parte, para poder afirmar su carácter teleológico, no basta establecer que no se ha empleado dicho recurso. Se necesita, además, determinar si la prioridad de lo correcto se ha garantizado utilizando el recurso configurativo. Si este recurso ha sido utilizado, estaremos en presencia de una teoría deontológica; de lo contrario, la teoría será teleológica.

La segunda razón por la que, a mi juicio, la reconstrucción de Dworkin parece equivocada se asocia a su manera de entender el recurso justificatorio. Dworkin piensa que, para determinar si tal recurso ha sido utilizado o no, hay que prestar atención exclusivamente al carácter de la premisa básica del argumento que conduce al principio de corrección o justicia. Si esta premisa es un derecho, entonces el argumento a favor del principio de justicia garantiza el respeto de la prioridad de lo correcto en relación con lo bueno. Si, por el contrario, establece meramente un determinado estado de cosas como valioso, entonces el argumento no garantiza que dicha prioridad sea respetada.

Kymlicka hace suyos los dos errores de Dworkin. Por un lado, sostiene que basta determinar si se ha utilizado o no el recurso justificatorio, para establecer el carácter deontológico o teleológico de una concepción de justicia. Por el otro, piensa que este recurso ha sido utilizado efectivamente - garantizando el carácter deontológico de la concepción - si la premisa básica de la que parte la justificación que conduce a los principios es un derecho. No obstante, aunque ambas afirmaciones son erróneas, sólo el error de la segunda es relevante a la hora de evaluar su argumento a favor de considerar al utilitarismo como una doctrina deontológica.

Si fuera posible mostrar que existe una justificación del utilitarismo cuya premisa básica es un derecho, y si esto bastara para determinar que el recurso justificatorio ha sido utilizado - garantizando la prioridad de lo correcto-, el objetivo de Kymlicka de brindar una interpretación deontológica del utili- 
tarismo se habría alcanzado, con independencia de si la primera afirmación - la que señala que las teorías que no utilizan el recurso justificatorio son teleológicas - es errónea o no.

Dicho en otras palabras: mostrar que el carácter teleológico de una concepción no puede determinarse constatando que no se ha utilizado el recurso justificatorio no es una objeción al argumento de Kymlicka a favor de una interpretación deontológica del utilitarismo.

En consecuencia, es necesario detenernos en la segunda de las tesis que Kymlicka comparte con Dworkin, y en la cual se basa su interpretación deontológica del utilitarismo. Kymlicka interpreta que basta que la premisa básica del argumento que conduce al principio utilitarista establezca un derecho - en lugar de establecer como bueno un determinado estado de cosaspara aseverar que se ha empleado el recurso justificatorio. Esto, a su vez, garantiza que la prioridad de lo correcto se haya cumplido y que la concepción de justicia resultante posea carácter deontológico.

Sostengo que tanto Kymlicka como Dworkin están equivocados. A mi criterio, no es suficiente que la premisa básica del argumento que conduce al principio utilitarista establezca el derecho a la igual consideración, para decir que se ha usado el recurso justificatorio y que se ha otorgado la prioridad a lo correcto. Como he señalado, lo que esta exigencia plantea es que en el argumento justificatorio no se introduzca una noción de "bien", definida de manera independiente a cualquier exigencia moral. Ahora bien, ¿se satisface esta exigencia con el argumento de Kymlicka? En mi opinión, la respuesta es no.

Como se recordará, el argumento estaba constituido por las siguientes afirmaciones:

a) Las personas tienen el derecho a ser tratadas como igualmente importantes.

b) Respetar la importancia de las personas implica tomar en consideración los intereses de todos por igual, a partir de lo cual se extrae la siguiente conclusión: 
c) Las acciones moralmente correctas son aquellas que desarrollan al máximo la utilidad.

Ahora bien, este argumento contiene algunas premisas implícitas que deben ser puestas de manifiesto para analizar su estructura. Una versión más completa del mismo señalaría:

a) Las personas tienen el derecho a ser tratadas como igualmente importantes.

b) Respetar la importancia de las personas implica tomar en consideración el "bien" de todos por igual.

c) El "bien" de cada uno consiste en su bienestar o en la "utilidad"; a partir de lo cual se concluye que:

d) Debe otorgarse igual peso al bienestar de cada persona, y de esto se sigue:

e) Las acciones moralmente correctas son aquellas que desarrollan al máximo el bienestar o la "utilidad" general.

La premisa (c) de este argumento a favor del principio utilitarista es la que lo vuelve incapaz de garantizar que se otorgue la prioridad de lo correcto en relación con lo bueno.

Lo bueno se define como el bienestar o la "utilidad", y en qué consiste esto se determina con independencia de cualquier criterio de corrección o estándar moral. La justificación que se ofrece del utilitarismo no garantiza la satisfacción de la prioridad de lo correcto porque, a diferencia de lo que sucede con la noción de bienes primarios, la noción de bienestar o utilidad no es de índole moral.

Refiriéndose a esto, Scanlon señala:

aunque la noción de bienestar es importante para la moralidad, no es en sí misma una noción moral. Representa lo que un individuo tiene razón para querer para sí, dejando de lado el interés por los otros y cualquier restricción u obligación moral. El bienestar es de este modo un ingrediente externo del pensamiento moral que no está ya configurado por suposiciones morales. (Scanlon 1998, p. 109) 
En consecuencia, el respeto de la prioridad de lo correcto no queda establecido por el hecho de que la premisa básica del argumento que Kymlicka presenta a favor del utilitarismo sea el derecho de los individuos a ser tratados con igual respeto y consideración. Sería necesario, además, que la noción de "bien" utilizada en el argumento estuviera configurada en función de ese derecho. Ahora bien, en el argumento de Kymlicka, el derecho a recibir la misma consideración explica por qué tomamos en cuenta el "bien" de todas las personas por igual (b), pero no explica por qué consideramos que su "bien" es equivalente a su bienestar o "utilidad" (c). Dicho de otra manera, el "derecho a recibir la misma consideración" es una razón para considerar el bien de todos por igual, pero en sí mismo no es la razón por la que se considera la "utilidad" o el bienestar como algo bueno. De esa forma, lo bueno se define con independencia de cualquier interés por los otros o al margen de cualquier restricción moral. Las consideraciones fundadas en el derecho de igual respeto no configuran ni limitan lo que se considera "útil". Por ese motivo, el argumento que Kymlicka ofrece a favor del utilitarismo no logra mostrar que esta doctrina satisfaga la condición de dar prioridad a lo correcto. ${ }^{13}$

\footnotetext{
${ }^{13}$ La otra cara del argumento de Kymlicka que intenta mostrar que, al igual que la justicia como equidad, el utilitarismo es una doctrina teleológica, es el argumento dirigido a establecer que la justicia como equidad es una doctrina de tipo utilitarista. Esto sería así — sostiene este argumento-, debido a que las preferencias que tienen las partes en la posición original, en cuanto representantes de ciudadanos libres e iguales dotados de los dos poderes morales fundamentales, se podrían representar en una función de utilidad. La réplica de Rawls se ha dirigido a señalar que el carácter utilitarista de una concepción no depende de que se use alguna función de utilidad en su formulación, sino de la manera en que se construye esa función. Si la función de utilidad está construida con base en una concepción normativa de persona, la cual establece que al momento de determinar qué normas van a regir nuestro trato recíproco debemos vernos como personas libres e iguales, entonces tal concepción no es utilitarista. $\mathrm{Si}$, por el contrario, la función de utilidad está construida con base en los intereses y preferencias actuales de los individuos, tal concepción es utilitarista. En el primer caso, la función de utilidad no representa una noción de bien independiente de cualquier exigencia moral, en el segundo sí. (Cfr. Rawls 2001, pp. 106-109.)
} 


\section{Configuración deontológica del principio utilitarista}

La segunda razón de Kymlicka para considerar que el utilitarismo es una concepción deontológica se vincula al recurso configurativo para garantizar la prioridad de lo correcto en relación con lo bueno. Para hacer uso de este recurso, como se recordará, el principio de justicia no debe ser un principio maximizador.

Kymlicka señala que, en la interpretación que él propone del utilitarismo, tal exigencia se satisface. Lo correcto se entiende como el tratar a las personas con igual respeto y consideración, y no como la maximización de lo bueno. El utilitarismo sería, simplemente, una manera de "desarrollar" o especificar ${ }^{14}$ esta idea. Afirma Kymlicka:

De acuerdo con esta interpretación del utilitarismo, el problema es cómo tratar equitativamente a personas distintas. La solución estándar consiste en dar el mismo peso a los intereses de cada persona. La vida de cada una importa igual desde el punto de vista moral, y por lo tanto los intereses de cada una merecen la misma consideración [...]. Ahora bien, esta idea de tratar a las personas con igual consideración es muy imprecisa y si en verdad queremos que sea una guía para nuestras acciones será necesario especificarla. Una manera obvia, y quizás inicialmente atractiva, de hacer esto es dar igual peso a cada preferencia de cada persona, sin tomar en cuenta el contenido de la preferencia o la situación material de la persona. (Kymlicka 1988, p. 177)

Ahora bien, si se interpreta de este modo, el argumento de Kymlicka resulta completamente inocuo en relación con el de Rawls. Kymlicka estaría afirmando, en realidad, que lo correcto es tratar a las personas con igual respeto y consideración. El procedimiento de decisión utilitarista sería solamente una manera de llevar a la práctica este criterio. Su argumento, entonces,

${ }^{14}$ He optado por traducir "to spell out", que Kymlicka utiliza, como "desarrollar" una idea. No estoy plenamente satisfecho con la palabra castellana escogida, pero considero que es la más adecuada. 
no tendría ninguna implicación con respecto al de Rawls, puesto que ambos se estarían refiriendo al utilitarismo en sentidos distintos. El argumento de Kymlicka se referiría al utilitarismo como un procedimiento de decisión, mientras que, como he señalado, las referencias de Rawls al utilitarismo - y, en consecuencia, el carácter teleológico que le atribuye- deben interpretarse a la luz de la concepción que afirma que el principio utilitarista es el criterio último de corrección. ${ }^{15}$

Por lo tanto, el argumento de Kymlicka debe entenderse, en mi opinión, como si señalara que el utilitarismo, en su interpretación deontológica, sostiene dos posiciones. La primera: que la acción correcta es aquella que maximiza la utilidad general; la segunda: que la posición igual de las personas debe ser respetada en el procedimiento de decisión que busca determinar cuál es la acción que maximiza la utilidad. Así, sostiene que, dada la interpretación que él brinda del utilitarismo, éste sería una doctrina

tan deontológica como cualquier otra, pues exige que, en el procedimiento decisorio, se respete a toda costa la posición igual de las personas. Si sus preferencias no han sido contadas de la misma manera, entonces las hemos tratado injustamente, no como iguales. (Kymlicka 1988, p. 178)

Para esclarecer la manera en que Kymlicka entiende el utilitarismo, detengámonos un momento y examinemos el siguiente ejemplo: supongamos que un dictador interesado sólo en satisfacer su deseo de poder, y sin considerar las preferencias de nadie más, adopta una medida de gobierno que a la postre tiene como resultado la maximización de la utilidad. Si ahora nos preguntamos si su acción ha sido correcta en función del principio

${ }^{15}$ Queda demostrado que ésta no es la interpretación adecuada del argumento de Kymlicka, a pesar del soporte textual que pueda obtener a partir de algunas de sus afirmaciones, por lo que él mismo señala en su libro Contemporary Political Philosophy. An Introduction: "El utilitarismo es esencialmente un "criterio de corrección', no un 'procedimiento decisorio" " (Kymlicka 1990, p. 29). Es decir, en este aspecto no existe desacuerdo alguno con Rawls. En su argumento, por lo tanto, el utilitarismo se debe interpretar como criterio de corrección. 
utilitarista, según Kymlicka, habría dos respuestas posibles, sea que se lo interprete como una doctrina teleológica o como una doctrina deontológica. La primera diría que su acción ha sido correcta, en cuanto el acto ha maximizado la utilidad general. La segunda sostendría que el acto ha sido incorrecto ya que, aunque se ha terminado maximizando la utilidad, en el procedimiento de decisión, las preferencias de las personas no se han tenido en cuenta por igual. En realidad, en el procedimiento de decisión del ejemplo, las preferencias no han sido tomadas en cuenta de manera alguna. Esta última sería la respuesta del utilitarismo deontológico propuesto por Kymlicka.

En esta interpretación, lo correcto es prioritario con respecto a lo bueno. La manera en que debe tratarse a las otras personas no es aquella en la que se maximiza el "bien", definido de forma independiente. Por el contrario, hay consideraciones fundadas en el respeto por los otros las cuales limitan la búsqueda de lo bueno. La acción correcta es aquella que maximiza el "bien", entendido como utilidad general, siempre que en el procedimiento de decisión se haya respetado el derecho de las personas a que sus preferencias sean contadas por igual.

Es imprescindible hacer una aclaración en relación con lo señalado. El razonamiento recién expuesto no puede interpretarse como si indicara que el principio utilitarista respeta la prioridad de lo correcto, debido a que una de las razones para adoptarlo es el interés en tratar a las personas con igual respeto y consideración. Dicho en otras palabras, el argumento no señala que el utilitarismo sea deontológico porque su premisa básica de justificación se base en el derecho de las personas a ser tratadas como iguales y a que sus preferencias sean contadas en el procedimiento decisorio de manera equitativa, puesto que las razones para afirmar que un principio es correcto no forman parte, necesariamente, del principio mismo. ${ }^{16}$

${ }^{16}$ En coincidencia con lo señalado, Rawls afirma: "Por lo tanto, aunque Brandt sostiene [...] que, desde un punto de vista filosófico, el mejor código es el que maximiza la utilidad media, no afirma que el principio de utilidad deba pertenecer al código mismo. De hecho, niega que dentro de la moralidad pública el tribunal de apelación definitiva tenga que ser la utilidad. 
El argumento debe interpretarse, entonces, como si señalara dos cosas: en primer lugar, que la premisa básica que justifica el principio de corrección utilitarista es el derecho a la igual consideración; y, en segundo lugar - y debido a lo anterior-, que el principio de corrección debe estar configurado para incluir, junto con la exigencia maximizadora del "bien", la exigencia de respetar el derecho de los ciudadanos a que sus preferencias sean contadas por igual en el procedimiento decisorio.

Ahora bien, aunque la afirmación de Kymlicka de que tal doctrina sea deontológica es verdadera, su afirmación de que sea utilitarista, en el sentido en que Rawls interpreta este término, es falsa.

Como hemos señalado, para Rawls, el utilitarismo es la doctrina que afirma que la acción correcta es aquella que permite obtener el saldo neto más alto de satisfacción de los individuos. Una concepción utilitarista es aquella que sostiene que éste es el criterio último de corrección. Pero, entonces, si una doctrina establece que el criterio de corrección es el de maximización de la utilidad, respetando el derecho de los ciudadanos a que sus preferencias sean contadas por igual en el procedimiento decisorio, el primero de estos requisitos ha dejado de ser el criterio último de corrección y, por lo tanto, tal doctrina no es ya una de tipo utilitarista. ${ }^{17}$

De este modo, según la definición dada en el texto, su visión no es utilitarista" (Rawls 1971, p. 182, n. 31 [159 n. 32]).

${ }^{17}$ Estaríamos en presencia de algo similar a lo que Rawls denomina una concepción mixta; sin embargo, no sería idéntica, puesto que todas estas concepciones aceptan el primer principio de las libertades básicas iguales, mientras que aquí lo único que se exigiría sería que se respetara por igual el derecho a que todas las preferencias sean consideradas sin distinción. No obstante, esto bastaría para que tal concepción no fuera tipo utilitarista. Señala Rawls, refiriéndose a las concepciones mixtas: "Ninguna de estas visiones es utilitarista, porque aun si el principio de utilidad sustituye al segundo principio, o a alguna parte del mismo, como el principio de la diferencia, la concepción de utilidad tiene todavía un lugar subordinado" (Rawls 1971, p. 316 [278]; las cursivas son mías). Es decir, no son utilitaristas, porque la exigencia de maximizar la utilidad no es el criterio último de corrección.

Por otra parte, ésta es la razón por la que, según Rawls, de las dos comparaciones en las que está organizado el razonamiento que conduce a los principios de justicia (la que contrapone el principio de las libertades básicas iguales y 
De esta manera, en su intento de brindar una interpretación deontológica del utilitarismo, Kymlicka se enfrenta a un dilema: o bien su interpretación mantiene que el criterio último de corrección es el principio utilitarista que manda maximizar la utilidad de los ciudadanos en general - sin ninguna referencia al derecho de las personas a que sus preferencias sean contadas por igual en el procedimiento decisorio - y fracasa en su intento de mostrarse como un tipo de deontologismo; o bien adosa al principio maximizador de la utilidad, como criterio último de corrección, la exigencia de que tal derecho sea respetado en el procedimiento decisorio, y fracasa en su intento de que ésta sea una concepción utilitarista.

\section{Conclusión}

Los dos argumentos esgrimidos por Kymlicka para mostrar que una interpretación deontológica del utilitarismo es posible son incorrectos. El primero, porque no basta que la premisa básica a partir de la cual se justifica el principio utilitarista sea el derecho de los individuos a ser tratados con igual respeto y consideración, para que el resultado sea una concepción que respete la prioridad de lo correcto en relación con lo bueno. Para que esto se diera, se necesitaría, además, que la noción de "bien" utilizada en el argumento estuviera configurada en función de este derecho. Como he señalado, tal exigencia no se satisface en el argumento ofrecido por Kymlicka, toda vez que la noción de utilidad no está configurada a partir de ninguna consideración moral o interés por los otros.

El segundo argumento también es errado, porque si se afirma que el principio de corrección mismo debe incluir - junto con la exigencia maximizadora del "bien" - la exigencia de respetar el

el principio de la diferencia con el principio utilitarista, y la que contrapone los dos principios con una concepción que acepta el primero, pero que, para distribuir el ingreso y la riqueza, adopta un criterio utilitarista en lugar del principio de la diferencia), la primera es la fundamental. Rawls sostiene: "La primera comparación es la más fundamental, porque el objetivo de la justicia como equidad es elaborar una concepción de justicia política alternativa a aquellas encontradas en el utilitarismo, el perfeccionismo y el intuicionismo" (Rawls 2001, p. 97). 
derecho de los ciudadanos a que sus preferencias sean contadas por igual en el procedimiento decisorio, la concepción resultante tiene carácter deontológico, pero no es ya una concepción de tipo utilitarista.

Creo que una de las razones por las que tal vez Kymlicka pensó que era posible brindar una interpretación deontológica del utilitarismo es que confundió lo que justifica la decisión de adherirse a una doctrina, con lo que justifica la doctrina misma. De tal modo, como señala Rawls, es posible que algunos filósofos hayan aceptado el principio utilitarista creyendo que era la interpretación correcta de la equidad; es decir, pensando que era la mejor manera de tratar a las personas como iguales. ${ }^{18}$ Esta creencia sería la que justificaría su decisión de adherirse al principio utilitarista de justicia, pero no alcanzaría para mostrar que el utilitarismo es una doctrina deontológica que entiende que lo correcto no es aquello que maximiza la utilidad, sino aquello que es debido a los otros en la medida en que son considerados imparcialmente. Dicho de otra manera, aunque algunas personas crean tener razones fundadas en su sentido de la justicia o de lo correcto para adherirse al principio utilitarista, esto no vuelve al utilitarismo una doctrina deontológica.

El intento de Kymlicka de presentar una interpretación deontológica del utilitarismo no tiene éxito. Aun en la interpretación ofrecida por éste, el utilitarismo sigue siendo una concepción teleológica.

En consecuencia, su tesis con respecto a la falta de relevancia de la clasificación de las doctrinas en deontológicas o teleológicas no puede encontrar fundamento en el hecho de que sea posible proporcionar una interpretación deontológica del utilitarismo - manteniendo sus mismas deficiencias en el nivel normativo-.

\footnotetext{
${ }^{18}$ Rawls señala: “Algunos filósofos han aceptado el principio utilitarista debido a que creían que la idea de un observador simpatético imparcial era la interpretación correcta de la imparcialidad. [... ] El error de la doctrina utilitarista es que confunde imparcialidad con impersonalidad" (Rawls 1971, pp. 189-190 [165-166]).
} 
No he ofrecido ningún argumento para mostrar la fecundidad de esta clasificación; sin embargo, he desarticulado una objeción cuyo objetivo era señalar su completa irrelevancia. Las consideraciones que he propuesto sirven simplemente para demostrar que no puede darse por concluido el debate acerca de si la estructura teleológica o deontológica de las concepciones filosófico-políticas es una de las causas de sus defectos en el nivel normativo. ${ }^{19}$

\section{BIBLIOGRAFÍA}

Brink, David, 1986, "Utilitarian Morality and the Personal Point of View", Journal of Philosophy, vol. 83, no. 8, pp. 417-438.

Dworkin, Ronald, 1977, Taking Rights Seriously, Harvard University Press, Cambridge, Mass.

Kymlicka, Will, 1990, Contemporary Political Philosophy. An Introduction, Clarendon Press, Oxford.

—_, 1988, "Rawls on Teleology and Deontology", Philosophy and Public Affairs, vol. 17, no. 3, pp. 173-190.

Nozick, Robert, 1974, Anarchy, State and Utopia, Basil Blackwell, Oxford.

Parfit, Derek, 1984, Reasons and Persons, Clarendon Press, Oxford. Pettit, Philip, 1997, "Reply to Baron and Slote", en M. Baron, P. Pettit, y M. Slote, Three Methods of Ethics, Blackwell, Oxford, pp. 252-265.

Pogge, Thomas W., 1989, Realizing Rawls, Cornell University Press, Ithaca/Londres.

Rawls, John, 2001, Justice as Fairness. A Restatement, Harvard University Press, Cambridge, Mass.

— 1971, A Theory of Justice, Harvard University Press, Cambridge, Mass. (Segunda edición revisada en inglés: 1999; versión en castellano: Teoría de la justicia, trad. María Dolores González, Fondo de Cultura Económica, México, 1979.)

${ }^{19}$ Agradezco la generosa atención que durante la elaboración de este artículo me brindó el doctor Ricardo Caracciolo. Sus críticas y sugerencias han servido para mejorarlo sustancialmente. También agradezco a los participantes del Seminario de Filosofía Política que dirijo en la Facultad de Derecho de la Universidad Nacional de Córdoba (Argentina) por sus agudas observaciones, las cuales pusieron a prueba mis ideas, e hicieron que las revisara en más de una ocasión. 
Scanlon, T.M., 1998, What We Owe to Each Other, Harvard University Press, Cambridge, Mass./Londres.

Slote, Michael, 1986, "Moderation, Rationality and Virtue", en S. McMurrin (comp.), The Tanner Lectures on Human Values, vol. VII, Cambridge University Press, Cambridge, Mass./Londres.

Recibido el 11 de julio de 2003; revisado el 30 de marzo de 2004; aceptado el 6 de mayo de 2004. 